\title{
Quasiconformal Isotopies
}

\section{Citation}

Earle, Clifford J., and Curtis T. McMullen. 1988. Quasiconformal isotopies. In Holomorphic Functions and Moduli I, ed. D. Drasin, C. J. Earle, F. W. Gehring, I. Kra, and A. Marden, Vol. 10, Mathematical Sciences Research Institute Publications, 143-154. New York: Springer.

\section{Permanent link}

http://nrs.harvard.edu/urn-3:HUL.InstRepos:9925394

\section{Terms of Use}

This article was downloaded from Harvard University's DASH repository, and is made available under the terms and conditions applicable to Other Posted Material, as set forth at http:// nrs.harvard.edu/urn-3:HUL.InstRepos:dash.current.terms-of-use\#LAA

\section{Share Your Story}

The Harvard community has made this article openly available.

Please share how this access benefits you. Submit a story.

\section{Accessibility}




\title{
Quasiconformal Isotopies
}

\author{
Clifford J. Earle, Curt McMullen \\ June 1986, Math Sciences Research Institute, Berkeley, CA.
}

\section{Introduction}

Let $\mathrm{X}$ be a hyperbolic Riemann surface or orbifold, possibly of infinite topological complexity. Let $\phi: X \rightarrow \mathrm{X}$ be a quasiconformal map. We show the following conditions are equivalent ( $\$ 1)$ :

(a) $\phi$ has a lift to the universal cover $\Delta$ which is the identity on $\mathbf{S}^{1}$;

(b) $\phi$ is homotopic to the identity rel the ideal boundary of $X$; and

(c) $\phi$ is isotopic to the identity rel ideal boundary, through uniformly quasiconformal maps.

(A related result in the PL category was established by Epstein [Eps].) The proof relies on the barycentric extension introduced by Douady and Earle [DE]. Applications include the equivalence of alternative definitions of Teichmüller space and new proofs of the Bers-Greenberg theorem and of the contractibility of $\operatorname{Diff}_{0}(\mathrm{X})$.

To understand the relation of condition (b) to other notions of relative homotopy, we study the geometry of the universal covering map $\pi: \Delta \rightarrow \mathrm{X}$. For a subdomain of the Riemann sphere, we show a homotopy rel the frontier of $\mathrm{X}$ is a homotopy rel ideal boundary (but the converse is false for certain non-locally connected domains) ( $\$ 2)$. We show a homotopy rel ideal boundary lifts to a homotopy rel the sphere at infinity (but this fails for hyperbolic 3-manifolds) (\$3). Finally, under the assumption of uniform quasiconformality, all notions of relative isotopy coincide. The proofs use harmonic measure and hyperbolic geometry.

These results have applications to the deformation theory of rational maps and Kleinian groups.

\section{$\S 1$. Riemann Surfaces and Orbifolds}

We will work in the category of hyperbolic orbifolds, in the interest of obtaining the BersGreenberg theorem.

Definitions. Let $\Gamma$ be a Fuchsian group, that is a discrete subgroup of conformal automorphisms of the unit disk $\Delta$; we do not require $\Gamma$ to be torsion-free or finitely generated. Then the quotient $X=\Delta \Gamma$ has the structure of a hyperbolic orbifold; each point of $\mathrm{X}$ has a neighborhood which is modelled on the quotient of a disk by a finite group of rotations. The projection $\pi: \Delta \rightarrow \Delta \Gamma=X$ is a covering map of orbifolds; it is the universal covering of the orbifold $\mathrm{X}$. Let $\mathrm{B} \subset \mathrm{X}$ denote the discrete set of branch points of $\mathrm{X}$, i.e. the points in the quotient corresponding to fixed points of elliptic elements of $\Gamma$. Then $\mathrm{X}-\mathrm{B}$ has the structure of an ordinary Riemann surface.

By definition, a continuous map $\phi: \mathrm{X} \rightarrow \mathrm{X}$ is a map which is covered by a continuous map on the universal cover of $X$. In other words there must exist a continous map $\hat{\phi}: \Delta \rightarrow \Delta$ such that $\phi^{\circ} \pi=\pi^{\circ} \hat{\phi}$. The map $\hat{\phi}$ is unique up to composition on the right and the left with elements of $\Gamma$.

We say $\phi$ is the identity on $\mathbf{S}^{1}$ if some choice of $\hat{\phi}$ can be completed to a continuous map of the closed disk $\Delta$ pointwise fixing its boundary $\mathbf{S}^{1}$.

We say $\phi$ is quasiconformal or conformal, if $\hat{\phi}$ is quasiconformal or conformal (this property is clearly independent of the choice of $\hat{\phi}$ ).

The authors are grateful for financial support from NSF grants DMS-8601016 (first author) and 8210790. 
Let $\Omega \subset \mathbf{S}^{1}$ denote the complement of the limit set of $\Gamma$; then the quotient $(\Delta \cup \Omega) \Gamma$ is an orbifold with interior $\mathrm{X}$ and boundary $\Omega \Gamma$, which we call the ideal boundary of $\mathrm{X}$ (denoted ideal- $\partial \mathrm{X}$ ).

Let I denote the unit interval [0,1], and consider a homotopy $\phi: \mathrm{I} \times \mathrm{X} \rightarrow \mathrm{X}$ (which we denote by $\phi(\mathrm{t}, \mathrm{x})$ or $\left.\phi_{\mathrm{t}}(\mathrm{x})\right)$, such that $\phi_{0}=\mathrm{id}$. By definition, such a homotopy is covered by a homotopy on the universal cover of $\mathrm{X}$.

Then $\phi_{\mathrm{t}}$ is a homotopy rel ideal boundary if it can be completed to a homotopy of $(\mathrm{X} \cup$ ideal- $\partial \mathrm{X})$ pointwise fixing the ideal boundary. Similarly $\phi_{\mathrm{t}}$ is a homotopy $\mathrm{rel} \mathbf{S}^{1}$ if it has a lift $\hat{\phi}_{\mathrm{t}}$ to a homotopy of the universal cover $\Delta$ of $\mathrm{X}$ which can be completed to a homotopy of $\Delta$ pointwise fixing $\mathbf{S}^{1}$.

Remark. It is clear that a homotopy rel $\mathbf{S}^{1}$ is a homotopy rel ideal boundary; the converse will be established in $\S 3$.

Theorem 1.1. Let $\phi: X \rightarrow X$ be a K-quasiconformal map. Then the following conditions are equivalent:

(a) $\phi$ is the identity on $\mathbf{S}^{1}$.

(b) $\phi$ is homotopic to the identity rel the ideal boundary of $\mathrm{X}$.

(c) $\phi$ is isotopic to the identity rel ideal boundary, through $\mathrm{K}^{\prime}$-quasiconformal maps (where K' depends only on $\mathrm{K}$ ).

\section{Remarks.}

(1) We shall see in $\S \S 2$ and 3 that for a uniformly quasiconformal isotopy, the conditions (i) bounded, (ii) rel $\mathbf{S}^{1}$, (iii) rel ideal boundary and (iv) rel frontier (for a subdomain of $\hat{\mathbf{C}}$ ) are all equivalent. Thus the isotopy of (c) enjoys all these properties.

(2) The isotopy that we will construct in the proof respects the symmetries of $\phi$. More precisely, let $\mathrm{G}$ be a group of conformal automorphisms of X, or more generally a semigroup of self-coverings of $\mathrm{X}$. Then if $\phi^{\circ} \mathrm{g}=\mathrm{g}^{\circ} \phi$ for all $\mathrm{g}$ in $\mathrm{G}$, the same will be true for each map $\phi_{\mathrm{t}}$ occuring in the isotopy. This is useful for applications to rational maps and Kleinian groups.

Proof of Theorem 1.1. The implications $(c) \Rightarrow(b) \Rightarrow$ (a) are easy to check.

To see (a) $\Rightarrow>$ (c), let $\Gamma$ be a Fuchsian group uniformizing $X$, and let $\phi: X \rightarrow X$ be a quasiconformal map which is the identity on $\mathbf{S}^{\mathbf{1}}$. Then $\phi$ has a lift $\psi: \Delta \rightarrow \Delta$ that extends continuously to the identity on $\mathbf{S}^{1}$ and commutes with every element of $\Gamma$.

Let $\mu$ denote the dilatation of $\psi$, and let $\alpha_{t}$ denote the unique quasiconformal map of the disk to itself with dilatation $t \mu$, fixing $(1, \mathrm{i},-1)$. By Ahlfors-Bers $[\mathrm{AB}], \alpha_{\mathrm{t}}$ gives an isotopy of $\bar{\Delta}$, but not necessarily rel $\mathbf{S}^{1}$. Since $\mu$ is $\Gamma$-invariant,

$$
\Gamma_{\mathrm{t}}=\alpha_{\mathrm{t}}^{-1} \Gamma^{\circ} \alpha_{\mathrm{t}}
$$

is a family of Fuchsian groups isomorphic to $\Gamma$. Also $\alpha_{0}=\alpha_{1}=\mathrm{id}$ on $\mathbf{S}^{1}$, so $\Gamma_{0}=\Gamma_{1}=\Gamma$.

Let $\beta_{t}=\operatorname{ex}\left(\alpha_{t}^{-1}\right)$ denote the barycentric extension of the boundary values of $\alpha_{t}^{-1}$ (see DouadyEarle [DE]). By [DE] these extensions are $\mathrm{K}^{\prime}$-quasiconformal for $\mathrm{K}^{\prime}$ depending only on $\mathrm{K}$; they depend continuously on $t$, so $\beta_{t}$ gives an isotopy of the closed disk; and by conformal naturality they conjugate the action of $\Gamma_{\mathrm{t}}$ to $\Gamma$ throughout the entire unit disk. The initial and terminal maps $\beta_{0}$ and $\beta_{1}$ are the identity since they are barycentric extensions of the identity.

Let $\psi_{\mathrm{t}}=\beta_{\mathrm{t}}{ }^{\circ} \alpha_{\mathrm{t}}$. Then $\psi_{\mathrm{t}}$ is $\Gamma$-equivariant, so it descends to an isotopy $\phi_{\mathrm{t}}: \mathrm{X} \rightarrow \mathrm{X}$ connecting the identity map to $\phi$. By construction $\beta_{\mathrm{t}}$ and $\alpha_{\mathrm{t}}^{-1}$ agree on $\mathrm{S}^{1}$, so $\psi_{\mathrm{t}}$ is an isotopy rel $\mathbf{S}^{1}$ (and hence rel ideal boundary).

The argument shows $\psi_{\mathrm{t}}$ is in fact compatible with with the group of all Möbius transformations commuting with $\psi$, justifying remark (2) above. 


\section{Applications and Refinements.}

(1) Recall Bers' construction of the Teichmüller space Teich(X) [B1]: Teich(X) consists of pairs $(Y, \alpha)$ such that $\alpha: X \rightarrow Y$ is a quasiconformal homeomorphism of orbifolds, modulo the equivalence relation $(\mathrm{Y}, \alpha) \sim(\mathrm{Z}, \beta)$ if there is a conformal map $\gamma: \mathrm{Y} \rightarrow \mathrm{Z}$ such that

$$
\phi=\beta^{-1} \circ \gamma^{\circ} \alpha: X \rightarrow X
$$

is the identity on $\mathbf{S}^{1}$.

By the theorem, if we replace this condition by the requirement: $\phi$ admits a uniformly quasiconformal isotopy to the identity rel ideal boundary, we obtain the same equivalence relation. Thus we have an equivalent definition of $\operatorname{Teich}(\mathrm{X})$ which is somewhat more intrinsic to $\mathrm{X}$.

(Actually Bers does not define the Teichmüller space of $\mathrm{X}$ when $\mathrm{X}$ has branch points; however Teich $(X)$ as defined above is always isomorphic to a space he does define, namely Teich $(\Gamma)$, where $\Gamma$ is a Fuchsian group uniformizing the orbifold X.)

(2) Using this remark, we have an independent demonstration of the Bers-Greenberg theorem [BG] (compare Marden [Mar] and Gardiner [Gar]):

Corollary 1.2 (Bers-Greenberg). Let $X$ be a hyperbolic orbifold with branch set $B$. Then the Teichmüller spaces Teich(X) and Teich(X-B) are canonically isomorphic.

Proof. Restriction from $\mathrm{X}$ to $\mathrm{X}-\mathrm{B}$ defines a canonical bijection from quasiconformal homeomorphisms with domain $\mathrm{X}$ to those with domain $\mathrm{X}$-B.

A uniformly quasiconformal isotopy to the identity on X-B can be completed to an isotopy of the underlying topological space of $\mathrm{X}$, which fixes $\mathrm{B}$ pointwise and is therefore an isotopy with respect to the orbifold structure. Conversely an isotopy to the identity on $\mathrm{X}$ fixes B pointwise and hence restricts to an isotopy on X-B.

The bordered Riemann surface $(\mathrm{X}-\mathrm{B}) \cup$ ideal $-\partial(\mathrm{X}-\mathrm{B})$ is canonically identified with $(\mathrm{X} \cup$ ideal- $-\partial \mathrm{X})-\mathrm{B}$. This can be demonstrated by factoring the universal covering of the hyperbolic Riemann surface X-B through the universal covering $\Delta \rightarrow \mathrm{X}$. Thus one of the isotopies is rel ideal boundary iff the other one is.

Using the isotopy definition of Teichmüller space described in point (1) above, we see two maps $\alpha$ and $\beta$ are equivalent in Teich $(X)$ if and only if their restrictions to $X-B$ are equivalent in Teich(X-B), so we have established the desired bijection.

(3) Let $\mathrm{X}$ be a compact Riemann surface of genus $\mathrm{g} \geq 2$ and $\Delta \rightarrow \mathrm{X}=\Delta \Gamma$ a universal covering. The ideal boundary of $\mathrm{X}$ is empty, and every diffeomorphism $\phi: X \rightarrow X$ is quasiconformal. By a theorem of Earle and Eells [EE2], the group Diff ${ }_{0}(\mathrm{X})$ of diffeomorphisms $\phi: \mathrm{X} \rightarrow \mathrm{X}$ homotopic to the identity (with its $\mathrm{C}^{\infty}$ topology) is contractible. By Theorem 1.1, Diff ${ }_{0}(\mathrm{X})$ consists of the diffeomorphisms $\phi$ that are the identity on $\mathbf{S}^{1}$. The isotopy $\phi_{\mathrm{t}}$ constructed above depends continuously on both $\mathrm{t}$ and $\phi$ and provides an explicit contraction of $\operatorname{Diff}_{0}(X)$ to the identity map.

(4) In general the complex dilatation of the isotopy $\phi_{t}$ constructed above need not vary continuously (in $L^{\infty}$ ) as a function of t. Here is a more indirect proof of the implication (a) $=>$ (c), which yields a stronger result:

Theorem 1.3. The isotopy in part (c) of Theorem 1.1 can be chosen so the complex dilatation $\mu_{t}$ of $\phi_{t}$ varies continuously in $\mathrm{M}(\mathrm{X})$, the unit ball in the Banach space of measurable Beltrami differentials on $\mathrm{X}$ with the $\mathrm{L}^{\infty}$ norm. 
Proof. In [DE] the barycentric extension is used to prove that the Teichmüller space of any Riemann surface or orbifold is contractible. By Earle and Eells [EE1], the map from $\mathrm{M}(\mathrm{X})$ to Teich(X) obtained by solving the Beltrami equation is a locally trivial fibration, and the contractibility of Teich(X) implies the contractiblility of the fiber F lying over (X,id).

By definition, a quasiconformal map $\phi: X \rightarrow X$ is the identity on $\mathbf{S}^{1}$ if and only if the complex dilatation $\mu(\phi)$ lies in $\mathrm{F}$. Let $\mu_{\mathrm{t}}$ be a path in F connecting 0 (the complex dilatation of the identity map) to $\mu$. Then the corresponding quasiconformal isotopy $\phi_{t}$ satisfies the conditions of (c), and the complex dilatation varies continuously by construction.

Remark. Since the complex dilatation of $\phi_{\mathrm{t}}$ varies continuously, the equation

$$
\phi=\phi_{1}=\phi_{1 / \mathrm{n}}{ }^{\circ}\left[\phi_{1 / \mathrm{n}}^{-1} \circ \phi_{2 / \mathrm{n}}\right]^{\circ} \cdots \cdot{ }^{\circ}\left[\phi_{\mathrm{n}-1 / \mathrm{n}}^{-1} \phi_{1}\right]
$$

exhibits a factorization of $\phi$ into quasiconformal maps of small dilatation (for $\mathrm{n}$ sufficiently large), each of which is the identity on $\mathbf{S}^{1}$.

\section{§ 2. Planar Domains}

We now specialize to the case where $\mathrm{X}$ is a subdomain of the Riemann sphere. To avoid confusion with the ideal boundary, we will refer to the topological boundary of $X \subset \hat{\mathbb{C}}$ as the frontier of $X$ (denoted $\partial \mathrm{X}$ ). We say $\phi_{\mathrm{t}}: \mathrm{X} \rightarrow \mathrm{X}$ is a homotopy rel frontier if it can be completed to a homotopy of the closure $\mathrm{X}$ fixing $\partial \mathrm{X}$ pointwise.

Let $\pi: \Delta \rightarrow \mathrm{X}$ denote the universal covering map. Our discussion of isotopies depends on a sort of uniform continuity for the inverse of $\pi$, which may be of interest in its own right.

Let $\gamma$ : I $\rightarrow \mathrm{X}$ be a path, $\hat{\gamma}$ a lift of $\gamma$ to a path in $\Delta$. Let $\operatorname{diam}(\gamma)$ denote the diameter of $\gamma(\mathrm{I})$ in the spherical metric, and diam $(\hat{\gamma})$ the diameter of the lift in the Euclidean metric on the disk.

Lemma 2.1. There is a function $\alpha(t) \rightarrow 0$ as $t \rightarrow 0$ such that

$$
\operatorname{diam}(\hat{\gamma}) \leq \alpha(\operatorname{diam}(\gamma))
$$

for all paths $\gamma$ in $\mathrm{X}$ and all choices of lifts $\hat{\gamma}$.

Remark. The lemma is really a comparison between metrics, which can be rephrased as follows. Let $\mathrm{p}$ $: \hat{X} \rightarrow X$ denote a topological universal covering for $\mathrm{X}$. Define the path metric $\mathrm{d}(\mathrm{x}, \mathrm{y})$ on $\hat{\mathrm{X}}$ by

$$
\mathrm{d}(\mathrm{x}, \mathrm{y})=\inf \{\operatorname{diam} \mathrm{p}(\gamma): \gamma \text { is a path joining } \mathrm{x} \text { to } \mathrm{y} \text { in } \hat{\mathrm{X}}\} \text {. }
$$

A choice of basepoints determines a homeomorphism $\pi^{-1}{ }^{\circ} \mathrm{p}: \hat{\mathrm{X}} \rightarrow \Delta$. The lemma states that this homeomorphism is uniformly continuous from the path metric to the Euclidean metric on the disk.

Using the Lemma above, we will prove

Theorem 2.2. A homotopy to the identity rel frontier is also a homotopy rel ideal boundary.

\section{Remarks.}

(1) This result is an important technical point in Sullivan's proof of the no wandering domains theorem for rational maps [Sul] and a parallel proof of Ahlfors' finiteness theorem [B3]. In those works less general versions of the above are established by quite different arguments.

(2) The converse of Theorem 2.2 is false. For example, consider a domain with a comb in the boundary, such as $\mathrm{X}=$ the upper half-plane with the vertical segments of unit length lying above $\mathrm{z}=$ $1,1 / 2,1 / 3, \ldots, 0$ removed. Define an isotopy on each rectangle $[1 /(n+1), 1 / n] \times[0,1]$ starting at the identity, fixing the boundary throughout, and moving some interior point a definite vertical distance (say 1/2). Extend by the identity to an isotopy of the whole region $X$. The result is an isotopy rel ideal 
Figure 2.1. An isotopy rel ideal boundary but not rel $\partial \mathrm{X}$.

boundary (since the diameters of pre-images of the rectangles in the universal cover $\Delta$ tend to zero in the Euclidean metric), but not rel $\partial \mathrm{X}$ (no continuous extension is possible near the vertical segment over $\mathrm{z}=0$ ). (See Figure 2.1.)

This example cannot be made quasiconformal. We say an isotopy is bounded if it moves points only a uniformly bounded distance in the Poincare' metric; more precisely, each path $\phi(\mathrm{I}, \mathrm{x})$ has a lift to $\Delta$ whose Poincare' diameter is bounded independent of $\mathrm{x}$.

\section{Proposition 2.3.}

(a) A K-quasiconformal isotopy rel $\mathbf{S}^{1}$ is a bounded isotopy.

(b) A bounded isotopy is an isotopy rel frontier.

Proof. (a) A K-quasiconformal map fixing the boundary of the unit disk moves points in the interior only a uniformly bounded distance in the Poincare' metric. (b) Since the ratio of Poincare' metric to spherical metric tends to infinity as one nears the frontier of $\mathrm{X}$, the isotopy extends continuously to the identity on $\partial \mathrm{X}$.

Corollary 2.4. Let $\phi: X \rightarrow X$ be K-quasiconformal. The following are equivalent.

(a) $\phi$ is the identity on $\mathbf{S}^{1}$.

(b) $\phi$ is homotopic to the identity rel the frontier of $X$.

(c) There is a $\mathrm{K}^{\prime}$-quasiconformal isotopy $\psi_{\mathrm{t}}$ of the whole Riemann sphere, such that $\psi_{\mathrm{t}}$ fixes $\hat{\mathbf{C}}-\mathrm{X}$ pointwise and its restriction to $\mathrm{X}$ provides an isotopy connecting $\phi$ to the identity.

Proof. Clearly (c) $\Rightarrow$ (b). By Theorem 2.2 the homotopy of (b) is also rel ideal boundary, so (b) $\Rightarrow$ (a). Now assuming (a), Theorem 1.1 yields a $\mathrm{K}^{\prime}$-quasiconformal isotopy $\phi_{\mathrm{t}}$ connecting $\phi$ to the identity. By Proposition 2.3 this extends to an isotopy rel frontier, which extends by the identity to an isotopy of the whole Riemann sphere. By a well-known result (see [B2] or [DH, Lemma 2]), the continuous extension of a quasiconformal map by the identity is still quasiconformal with the same dilatation, establishing (c).

We give the proof of 2.2 assuming the lemma on diameter of paths, to motivate the latter. 
Proof of Theorem 2.2. Let $\phi_{t}: \bar{X} \rightarrow \bar{X}$ be an isotopy connecting $\phi$ to the identity through maps fixing $\partial \mathrm{X}$. Let $\hat{\phi}_{\mathrm{t}}$ be the unique lift to an isotopy of $\Delta$ connecting a lift of $\phi$ to the identity. We claim $\hat{\phi}_{\mathrm{t}}$ can be completed to an isotopy of $\Delta$ fixing $\mathbf{S}^{1}$.

It suffices to construct for each $\varepsilon>0$ a neighborhood $U$ of $\mathbf{S}^{1}$ such that diam $\hat{\phi}(\mathrm{I}, \mathrm{z})<\varepsilon$ for all $\mathrm{z}$ in $\mathrm{U}$. To this end choose a neighborhood $\mathrm{V}$ of $\partial \mathrm{X}$ such that diam $\phi(\mathrm{I}, \mathrm{x})<\delta$ for all $\mathrm{x}$ in $\mathrm{V}$, where $\alpha(\delta)<\varepsilon$ and $\alpha$ is the function provided by Lemma 2.1. Such a neighborhood exists because the isotopy fixes $\partial \mathrm{X}$.

If $\pi(\mathrm{z})$ lies in $\mathrm{V}$, then $\operatorname{diam}(\hat{\phi}(\mathrm{I}, \mathrm{z}))<\varepsilon$ since it covers the path $\phi(\mathrm{I}, \mathrm{X})$. On the other hand, $\mathrm{X}-\mathrm{V}$ is a compact set, so if $\pi(\mathrm{z})$ lies in $\mathrm{X}-\mathrm{V}$ the Poincare' diameter of $\hat{\phi}(\mathrm{I}, \mathrm{z})$ is bounded above by a constant depending only on V. Since the Poincare' metric tends to infinity relative to the Euclidean metric as $\mathrm{z}$ tends to $\mathbf{S}^{1}$, there is a neighborhood $\mathrm{U}$ of $\mathbf{S}^{1}$ such that diam $\hat{\phi}(\mathrm{I}, \mathrm{z})<\varepsilon$ even if $\pi(\mathrm{z})$ lies in $\mathrm{X}-\mathrm{V}$.

It follows that $\hat{\phi}_{t}(\mathrm{z})$ tends to the identity uniformly as $|\mathrm{z}| \rightarrow 1$, and so may be completed to an isotopy of $\Delta$ fixing $\mathbf{S}^{1}$. Thus $\phi_{\mathrm{t}}$ itself is an isotopy rel ideal boundary.

Proof of Lemma 2.1. Let $\pi: \Delta \rightarrow \mathrm{X}$ denote the universal covering map. We may assume $\mathrm{X}$ $\subset \hat{\mathbf{C}}-\{0,1, \infty\}$ by applying a Möbius transformation (this changes spherical diameters by only a bounded factor.) Let $\mathrm{w}=\pi(0)$ and let $\lambda: \Delta \rightarrow \hat{\boldsymbol{C}}-\{0,1, \infty\}$ be the universal covering map normalized so that $\lambda(0)=w$. Then we may factor the map $\pi$ as $\pi=\lambda^{\circ} \alpha$, where $\alpha: \Delta \rightarrow \Delta$ and $\alpha(0)=0$.

Let $\gamma$ be a path in $X, \hat{\gamma}$ a lift of $\gamma$ to $\Delta$. We wish to show that $\operatorname{diam}(\hat{\gamma})$ is controlled by $\operatorname{diam}(\gamma)$. This is clear when $\gamma$ lies in a closed ball B centered at $\mathrm{w}$ and contained in $\mathrm{X}$; in fact all branches of $\pi^{-1}$ have uniformly bounded derivative throughout $\mathrm{B}$, so in this case we have $\operatorname{diam}(\hat{\gamma})=\mathrm{O}(\operatorname{diam}(\gamma))$.

Now assume $\gamma$ is a definite distance from $w$. Let $\gamma_{0}$ be the lift $\alpha(\hat{\gamma})$ of $\gamma$ to $\Delta$ via $\lambda$. For E a closed subset of the unit disk, let $\omega(\mathrm{z}, \mathrm{E})$ denote the harmonic measure of $\mathrm{E}$, i.e. the unique harmonic function on $\Delta-\mathrm{E}$ with boundary values 1 on $\mathrm{E}$ and 0 elsewhere. Equivalently, $\omega(\mathrm{z}, \mathrm{E})$ is the probability that a random path initiated at $\mathrm{z}$ hits $\mathrm{E}$ before exiting the unit disk.

To complete the proof, it suffices to establish the following chain of inequalities:

(i) $\operatorname{diam}(\hat{\gamma}) \leq \mathrm{O}(\omega(0, \hat{\gamma}))$

(ii) $\omega(0, \hat{\gamma}) \leq \omega\left(0, \gamma_{0}\right)$

(iii) $\omega\left(0, \gamma_{0}\right) \leq \mathrm{O}(\log (1 / \operatorname{diam}(\gamma)))$

since together they imply that the size of $\hat{\gamma}$ is controlled by the size of $\gamma$.

To see that (i) is plausible, one can argue that the probability of hitting a set of given diameter is least when the set is near the boundary of the disk, and the inequality is clear for a subset of the boundary. For a precise argument and a sharper inequality, we refer to the paper of Fitzgerald, Rodin and Warschawski [FRW].

Inequality (ii) follows from standard monotonicity properties of harmonic measure; there are more paths leading to $\gamma$ on the triply punctured sphere than on $X$, and any one in the correct homotopy class to hit $\hat{\gamma}$ is also in the correct homotopy class to hit $\gamma_{0}$. More formally, (ii) follows from the maximum principle, by comparing the boundary values of $\omega(\mathrm{z}, \hat{\gamma})$ and $\omega\left(\alpha(\mathrm{z}), \gamma_{0}\right)$ on $\Delta-\alpha^{-1}\left(\gamma_{0}\right)$.

Finally, inequality (iii) follows from a direct computation on the triply punctured sphere. The derivative of any branch of $\lambda^{-1}$ (from the spherical to Euclidean metric) is $\mathrm{O}(1 / \mathrm{d}$ ), where $\mathrm{d}$ is the dis-

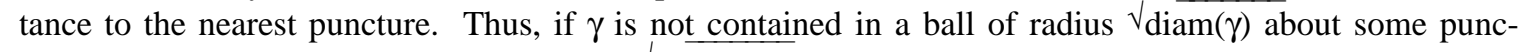

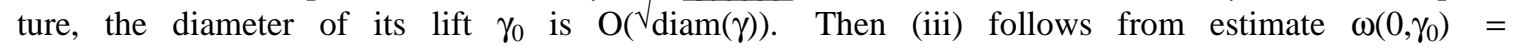
$\mathrm{O}\left(1 / \log \left(1 / \operatorname{diam}\left(\gamma_{0}\right)\right)\right)$, which holds for any path in the unit disk which is a definite distance from zero.

On the other hand, if $\gamma$ is contained in ball of radius $\sqrt{ }_{\operatorname{diam}(\gamma) \text { centered at a puncture, then its lift }}$ $\gamma_{0}$ is contained in a horoball of diameter $\mathrm{O}(1 / \log (1 / \operatorname{diam}(\gamma)))$, and (iii) follows from the fact that the harmonic measure of a horoball is comparable to its diameter. 


\section{Remarks.}

(1) It follows from the proof that we may take $\alpha(t)=\mathrm{O}(1 / \log (1 / \mathrm{t}))$ ) (and this order of magnitude is sharp for the triply punctured sphere).

(2) If $\mathrm{X}$ is simply connected we may take $\alpha(\mathrm{t})=\mathrm{O}\left(\sqrt{ }_{\mathrm{t}}\right)$ (and this is sharp for the complemement of a slit). To improve the estimate in the simply connected case, one applies the Beurling projection theorem (see Ahlfors [Ahlf]) to replace (ii) and (iii) with the inequality $\omega(0, \hat{\gamma}) \leq \mathrm{O}(\sqrt{ } \operatorname{diam}(\gamma))$. We are grateful to Peter Jones for a discussion of this point.

\section{§ 3. Ideal Boundary}

We conclude with a parallel discussion in which the frontier of $\mathrm{X}$ is replaced by its ideal boundary.

Let $\bar{X}=\mathrm{X} \cup$ ideal- $\partial \mathrm{X}$ denote the orbifold obtained by adjoining to $\mathrm{X}$ its ideal boundary. Let $\mathrm{K}_{1} \subset \mathrm{K}_{2} \subset \cdots$ denote an exhaustion of $\bar{X}$ by connected compact sets. Let $\gamma$ denote a path in $\mathrm{X}$ and $\hat{\gamma}$ a lift to the universal cover $\Delta$. Then the analogue of Lemma 2.1 becomes:

Lemma 3.1. There exists a sequence $\alpha(\mathrm{n}) \rightarrow 0$ as $\mathrm{n} \rightarrow \infty$, such that

$$
\operatorname{diam}(\hat{\gamma}) \leq \alpha(\mathrm{N}(\gamma))
$$

where $\mathrm{N}(\gamma)$ is the largest integer $\mathrm{N}$ such that $\gamma$ is disjoint from $\mathrm{K}_{\mathrm{N}}$.

Corollary 3.2. A homotopy to the identity rel ideal boundary is a homotopy rel $\mathbf{S}^{1}$ (and vice versa).

Proof. We mimic the proof of Theorem 2.2. Let $\phi_{t}$ be a homotopy rel ideal boundary, $\hat{\phi}_{t}$ the unique lift to a homotopy of $\Delta \cup \Omega$ such that $\hat{\phi}_{0}=$ id. We need only construct a neighborhood $U$ of $\mathbf{S}^{1}$ such that $\operatorname{diam}(\hat{\phi}(\mathrm{I}, \mathrm{z}))$ is less than $\varepsilon$ for all $\mathrm{z}$ in $\mathrm{U}$.

Choose $N$ such that $\alpha(N)$ is less than $\varepsilon$, and choose $M$ such that $K_{M}$ contains $\phi\left(I, K_{N}\right)$. Then $\operatorname{diam}(\hat{\phi}(\mathrm{I}, \mathrm{z}))<\varepsilon$ whenever $\pi(\mathrm{z})$ lies outside $\mathrm{K}_{\mathrm{M}}$.

Let $\mathrm{L} \subset \Delta \cup \Omega$ be a compact set such that $\pi(\mathrm{L})=\mathrm{K}_{\mathrm{M}}$. Then

$$
\hat{\phi}(\mathrm{I}, \gamma \mathrm{L})=\gamma(\hat{\phi}(\mathrm{I}, \mathrm{L})) \quad \text { for all } \gamma \text { in } \Gamma
$$

and since $\hat{\phi}(\mathrm{I}, \mathrm{L})$ is a compact subset of the domain of discontinuity, the Euclidean diameters of its translates under $\Gamma$ tend to zero. Thus $\operatorname{diam}(\hat{\phi}(\mathrm{I}, \mathrm{z}))<\varepsilon$ except possibly when $\mathrm{z}$ lies in one of finitely many translates of L. But these form a compact set, and the isotopy fixes $L \cap S^{1}$, so there is a neighborhood of $\mathbf{S}^{1}$ on which $\operatorname{diam}(\hat{\phi}(\mathrm{I}, \mathrm{z}))<\varepsilon$.

Remark. These two results fail for hyperbolic three-manifolds. For example, take a totally degenerate limit of quasifuchsian groups, such that the domain of discontinuity $\Omega$ is simply connected and the quotient $\mathrm{S}=\Omega \Gamma$ is a compact Riemann surface. Then the quotient three-manifold plus ideal boundary $\mathrm{X}$ is homeomorphic to a product $\mathrm{S} \times[0, \infty)$ (see Thurston [Thur] and Bonahon [Bon]). Thus the homotopy class of the closed geodesic corresponding to some fixed loxodromic element in the group is represented by loops arbitrarily far out in the end of X. A path wrapping many times around such a loop has a lift which nearly connects the fixed points of the loxodromic element, so its diameter does not go to zero.

A similar argument gives an isotopy rel ideal boundary which does not lift to an isotopy rel $\mathbf{S}^{2}$. (By a straightforward extension of Proposition 2.3, such an isotopy cannot be uniformly quasiconformal.) 
Proof of Lemma 3.1. It suffices to show, for $\varepsilon>0$, there exists an $N$ such that $\operatorname{diam}(\hat{\gamma})<\varepsilon$ whenever $\gamma$ lies outside $\mathrm{K}_{\mathrm{N}}$.

Let $\bar{X}=(\Delta \cup \Omega) \Gamma$. The union of $\Omega$ and the hyperbolic fixed points of $\Gamma$ is a dense subset of $\mathbf{S}^{1}$; choose a finite subset $F$ which is $\varepsilon$ - dense. Let $L$ denote the hyperbolic convex hull of $F$. Then $L / \Gamma$ is a compact subset of X. (After taking the quotient, the parts of $\mathrm{L}$ near $\mathbf{S}^{1}$ either touch the ideal boundary or spiral around the closed geodesics corresponding to the hyperbolic fixed points).

Thus for $\mathrm{N}$ sufficiently large, $\mathrm{L} \Gamma \subset \mathrm{K}_{\mathrm{N}}$. The components of $\overline{\Delta-\mathrm{L}}$ have diameter less than $\varepsilon$ (remark: it is here the proof breaks down in higher dimensions). If $\gamma$ lies outside $\mathrm{K}_{\mathrm{N}}$, its lift $\hat{\gamma}$ lies outside $\mathrm{L}$, so its diameter is less than $\varepsilon$, completing the proof.

\section{References}

[Ahlf] L. Ahlfors. Conformal Invariants. McGraw-Hill, 1973.

[AB] L. Ahlfors, L. Bers. Riemann mapping theorem for variable metrics. Annals of Math 72 (1960), pp.385-404.

[B1] L. Bers. Uniformization, moduli and Kleinian groups. Bull. London Math. Soc. 4 (1972), pp.257-300.

[B2] L. Bers. The moduli of Kleinian groups. Russian Math Surveys 29 (1974), pp.88-102.

[B3] L. Bers. On Sullivan's proof of the finiteness theorem and the eventual periodicity theorem. Preprint.

[BG] L. Bers, L. Greenberg. Isomorphisms between Teichmüller spaces. In Advances in the Theory of Riemann Surfaces, Princeton: Annals of Math Studies 66 (1971), pp.53-79.

[Bon] F. Bonahon. Bouts des varietes hyperbolique de dimension trois. To appear.

[DE] A. Douady, C. Earle. Conformally natural extension of homeomorphisms of the circle. To appear, Acta Mathematica.

[DH] A. Douady, J. Hubbard. On the dynamics of polynomial-like mappings. Ann. sci. Ec. Norm. Sup. 18 (1985), pp.287-344.

[EE1] C. Earle, J. Eells. On the differential geometry of Teichmüller spaces. J. Analyse Math. 19 (1967), pp.35-52.

[EE2] . A fibre bundle description of Teichmüller theory. J. Diff. Geom. 3 (1969), pp.19-43.

[Eps] D.B.A. Epstein. Curves on 2-manifolds and isotopies. Acta Math. 115 (1966), pp.83-107.

[FRW] C.H. Fitzgerald, B. Rodin, S.E. Warschawski. Estimates for the harmonic measure of a continuum in the unit disk. Trans. AMS 287 (1985), pp.681-685.

[Gar] F. Gardiner. A theorem of Bers and Greenberg for infinite dimensional Teichmüller spaces. These proceedings. 
[Mar] A. Marden. On homotopic mappings of Riemann surfaces. Annals of Math. 90 (1969), pp.18.

[Sul] D. Sullivan. Quasiconformal homeomorphisms and dynamics I: Solution of the Fatou-Julia problem on wandering domains. Annals of Math. 122 (1985), pp.401-418.

[Thur] W. Thurston. Geometry and Topology of Three Manifolds. Princeton lectures notes. 